\title{
Robotic Sacral Colpopexy Using Autologous Fascia Lata Compared
}

\section{to Mesh}

Megan E. Bock, MD

Department of Urology, Indiana University School of Medicine

535 North Barnhill Drive, Suite 150

Indianapolis, IN 46202

317.278 .0221

megbock@iu.edu

Ramzy Nagle, MD

Department of Urology, Indiana University School of Medicine

$\underline{\text { ranagle@iu.edu }}$

Mary Soyster, MD

Department of Urology, Indiana University School of Medicine

msoyster@iu.edu

Leo Song

Indiana University School of Medicine

songhaj@iu.edu

Isamu Tachibana, MD

Department of Urology, Indiana University School of Medicine isatachi@iupui.edu 
Jon K Hathaway, MD, PhD

Associate Professor of Obstetrics and Gynecology

Indiana University School of Medicine

jhathawa@iuhealth.org

Charles R. Powell, MD

Associate Professor of Urology

Indiana University School of Medicine

crpowell@iupui.edu 
Abstract

Objective

The objective is to compare robotic sacral colpopexy (RSC) utilizing autologous fascia lata to RSC with synthetic mesh in the treatment of pelvic organ prolapse.

Methods

We performed a prospective non-randomized case comparison trial at a single institution. We compared RSC utilizing either synthetic mesh or autologous fascia lata in women with symptomatic pelvic organ prolapse, stages II through IV. The primary outcome was anatomic prolapse recurrence determined by the Pelvic Organ Prolapse Quantification (POP-Q) exam. Secondary outcomes included patient reported outcomes such as the Urogenital Distress Inventory-6 (UDI-6) and Incontinence Impact Questionnaire-7 (IIQ-7). Complications were also recorded and categorized using the Clavien-Dindo system (CD). The hypothesis is that autologous fascia lata would provide equivalent anatomic and patient reported outcomes compared to mesh while eliminating mesh related complications.

Results

Sixty-four women underwent RSC with 19 (29.7\%) receiving fascia lata graft. The overall operative time was greater in the fascia lata group with mean fascia lata harvest time of 24.8 \pm 7.4 minutes. Intragroup comparisons of the fascia and mesh groups demonstrated significant improvement in pelvic measurements as well as patient reported outcomes. Intergroup comparison demonstrated equivalent success rates at $12.1 \pm 8.7$ months follow up. There was one apical failure in the fascia lata RSC group, however the difference was not statistically significant $(p=0.30)$. Significant complications in the fascia lata harvest group included two CD-II and one CD-IIIb. In the mesh group there was one mesh erosion requiring surgical excision (CD-IIIb). 
Conclusion

This is the first comparison between RSC with autologous fascia lata vs. mesh. Short-term anatomic outcomes were similar with autologous fascia lata use without the risk of mesh erosion. Morbidity from graft harvest site was not trivial. These results emphasize the need for a randomized controlled trial. 
Introduction

Pelvic floor disorders include a variety of clinical conditions including pelvic organ prolapse (POP), urinary incontinence, and fecal incontinence. ${ }^{1}$ POP includes anterior vaginal prolapse, apical or uterine descent, and posterior vaginal prolapse. ${ }^{1} \mathrm{POP}$ is a common problem amongst the aging female population and its prevalence is steadily increasing. POP, when defined by symptoms, has a prevalence of 3-6\%, but when based upon vaginal examination can be as prevalent as $50 \%$ in the female population. ${ }^{2}$ It is predicted that the number of women with at least one pelvic floor disorder will increase from 28.1 million in 2010 to 43.8 million by $2050 .^{3}$

Sacral colpopexy is considered the most effective and durable treatment for advanced apical prolapse. ${ }^{4}$ The goal is to resuspend the vagina to its anatomically correct position by securing the vaginal apex to the sacrum. This can be approached using a variety of techniques, including the use of either autologous tissue or mesh. Latini and Kreder were the first to report on autologous fascia lata. ${ }^{5}$ Years later, robotic sacral colpopexy (RSC) has been established as a safe and effective approach. RSC has been shown to have similar long term outcomes when compared to the open abdominal approach, with failure rates of $4.2-12 \%$ depending on how failure is defined. ${ }^{6,7}$ Previous studies have demonstrated a longer operative time for RSC, but decreased length of stay and blood loss. ${ }^{8}$ The number of RSC procedures has increased considerably in North America in recent years, making it the fastest growing approach for sacral colpopexy. ${ }^{9}$

Mesh is considered the gold standard option for apical prolapse, with fewer reported failures than tissue based repairs. ${ }^{10}$ The main complications associated with mesh use include suture and mesh erosion. ${ }^{11}$ With current Food and Drug Administration (FDA) warnings and patient safety concerns regarding vaginal mesh, patients and surgeons have a renewed interest in developing alternative mesh-free solutions. Autologous fascia RSC has been successful with minimal apical prolapse recurrences in the short term, within a small series. ${ }^{12}$ To date, no comparison study has been published regarding the comparative efficacy and safety of mesh versus autologous fascia during RSC. We hope to 
fill a critical need in the literature, given the prevalence of POP and the rising popularity of RSC $^{9}$

We present our recent experience with mesh and autologous fascia lata during RSC at a single institution by a single Female Pelvic Medicine and Reconstructive (FPMRS) fellowship trained surgeon. We report the operative and postoperative outcomes associated with mesh compared to autologous fascia lata. The hypothesis is that although the complications and operative times will likely differ, fascia lata will be non-inferior to mesh and will avoid mesh related complications.

\section{Materials and Methods}

This study was approved by the Institutional Review Board of Indiana University. A prospective non-randomized case comparison trial was designed and conducted at a single institution between November 2017 and December 2019. Eligible patients included women undergoing RSC with or without concomitant supracervical hysterectomy and/or urethral sling. All patients who underwent RSC during the study period were analyzed. The technique has been previously described. ${ }^{11,13}$

Patients were not randomized. The decision to use mesh versus autologous fascia lata was the result of an informed discussion and shared decision making between the physician and patient during preoperative counseling. The associated risks and benefits unique to each were described, including the FDA black box warnings $(2008,2011)$ regarding utilization of mesh as well as the risk of seroma, hematoma, thigh numbness, pain, muscle bulge, and DVT associated with fascia lata. ${ }^{14}$ The fascia was primarily harvested from the right lower extremity unless the patient had prior lower extremity surgery or specific laterality request.

Patient data was extracted from medical records and patient surveys. Data analyzed included preoperative demographics, operative timing, postoperative hospital stay, surgical complications, Pelvic Organ Prolapse Quantification (POP-Q) exam, and patient reported outcomes including Urogenital Distress Inventory-6 (UDI-6) and Incontinence Impact Questionnaire-7 (IIQ-7). ${ }^{15}$ 
POP-Q stage was defined according to standard criteria as defined by the International Continence Society and American Urogynecologic Society. ${ }^{16}$ Recurrence was defined as $>0$ in any compartment on postoperative POP-Q exam ( $\mathrm{Aa}, \mathrm{Ba}, \mathrm{Ap}, \mathrm{Bp})$. Recurrence at the apex was defined as $\geq 2 \mathrm{~cm}$ of apical descent as determined by point $\mathrm{C}$ relative to total vaginal length. Complications were categorized using the Clavien-Dindo (CD) classification system. ${ }^{17}$

Harvest of the Fascia Lata graft

The surgical technique has been previously described. ${ }^{19} \mathrm{~A}$ key element is to begin harvesting at least $5 \mathrm{~cm}$ cephalad and lateral to the patella after identifying Gerdy's Tubercle on the anterolateral aspect of the tibia, the point at which the tensor fascia lata coalesces into the iliotibial band. The longitudinal integrity of the ileotibial band must never be compromised making its identification crucial (Figure 1). Using malleable retractors and blunt dissection a sizeable graft can be harvested from a $4 \mathrm{~cm}$ incision. The graft can then be fashioned in a Y-configuration or sling using non-absorbable 0monofilament (Figures 2,3).

\section{Statistical Analysis}

Statistical comparisons were principally performed preoperatively and at the most recent post-operative visit for each patient using paired t-test. All statistical analyses were conducted with STATA SE version 16 (College Station, TX) as well as Microsoft Excel (Microsoft, Redmond, WA). Continuous variables were summarized using means and medians. Categorical variables were summarized using frequencies and percentages. Continuous variables were analyzed using 2 tailed t-test and categorical variables were analyzed using Fisher's exact test. A p-value less than 0.05 was considered statistically significant.

\section{Results}

\section{Baseline Characteristics}

A total of 64 women elected to undergo RSC from 2017 through 2019. Nineteen (29.7\%) elected autologous fascia lata RSC and 45 (70.3\%) chose mesh RSC. Baseline clinical 
characteristics were similar between the surgical groups and are summarized in Table 1. Women who elected to undergo fascia lata graft harvest tended to be younger (mean age $59.3 \pm 12.9$ years vs. $67.9 \pm 10.7$ years, $p=0.01)$. Gravidity was 2.3 vs. $3.3(p=0.03)$, parity was 2.1 vs. $3.0(p=0.02)$, and the majority were by vaginal delivery (1.8 vs. $2.9, p=0.01$ ). Women receiving autologous grafts were more likely to be diabetic $(p=0.01)$, but no difference was seen with regard to BMI or smoking status. Women receiving autologous grafts were also more likely to receive concomitant fascia slings $(p<0.001)$, and those undergoing mesh SCP were more likely to undergo mesh sling instead of fascia sling, but there were no significant differences between the two groups regarding other concomitant procedures including supracervical or total hysterectomy, anterior or posterior colporrhaphy, or enterocele repair. Pooled analysis of either concomitant midurethral or fascia sling in women receiving autologous grafts vs. mesh demonstrated no difference.

Symptomatically, patients undergoing mesh vs. fascia lata had no difference in preoperative frequency, urgency, or incontinence (Table 1). There were no significant differences in patient-reported UDI-6 and IIQ-7 surveys. Preoperatively, pelvic measurements according to POP-Q exam were similar in all categories as well as POP-Q stage. At the time of surgery, all patients had $\geq 2 \mathrm{~cm}$ apical descent with median POP-Q stage of 3 in both groups.

\section{Operative Details}

Operative details are outlined in Table 2. Eight of 19 (42.1\%) underwent concomitant robotic supracervical hysterectomy in the fascia lata group compared to $16 / 45$ (35.6\%) in the mesh group ( $p=0.78$ ). The average operative time for RSC with fascia was $288 \pm 39$ minutes compared to $237 \pm 31$ minutes for mesh $(p<0.001)$. Harvesting fascia required a mean of $24.8 \pm 7.4$ minutes. There were $6(31.6 \%)$ patients in the fascia group who underwent concomitant pubovaginal autologous sling and $8(17.8 \%)$ patients in the mesh group who underwent concomitant mid-urethral mesh sling. Both mesh and fascia were secured to the vaginal wall using a running delayed absorbable 2-0 monofilament suture with three non-absorbable 0 monofilament sutures for sacral fixation (range 2-4) as 
described previously. ${ }^{13}$ The average hospital stay for both groups was $1.0 \pm 0.7$ days. There was no difference between the two groups regarding estimated blood loss and postoperative urinary retention requiring a foley catheter. There were no patients requiring catheterization at the one month-mark.

For autologous fascia lata harvest, the average length of the graft was $18.3 \pm 2.5 \mathrm{~cm}$ and width was $4.2 \pm 1.4 \mathrm{~cm}$ (see Figure 1).

\section{Postoperative Outcomes}

Intra-group outcomes demonstrated postoperative improvement on all clinical and patient reported outcomes for fascia lata (Table 3) and mesh (Table 4). An inter-group comparison of clinical and patient reported outcomes is displayed in Table 5. Overall patient reported outcomes were similar between fascia lata and mesh. There was increased urinary frequency in the mesh group $(p=0.03)$ and increased de novo stress incontinence seen in the fascia lata group $(p=0.02)$. With regard to patient-reported outcomes, there was no difference seen on the UDI-6, but an increased total score in the fascia lata group on the IIQ-7 ( $p=0.05)$. Anatomic outcomes were not significantly different between groups. The vast majority of patients in both groups exhibited POP-Q stage 2 or less (94.8\% in fascia and $97.8 \%$ in mesh). One patient in the fascia lata group exhibited failure in both the anterior and apical compartments. The mesh group had five recurrences in the posterior compartment and one failure in the anterior compartment at a combined mean follow-up of $12.1 \pm 8.2$ months. The differences in recurrence were not statistically significant (Table 5).

\section{Postoperative Complications}

In the fascia lata group, one patient developed acute tubular necrosis which resolved with intravenous fluid resuscitation (CD-II), one developed unilateral hydronephrosis requiring ureteral stent and balloon dilation of the affected ureter which is resolved on follow up ultrasound 3 months later (CD-IIIb), one hematoma at the harvest site requiring transfusion (CD-II), and one ipsilateral DVT (CD-II). In the mesh group, one mesh erosion was noted requiring vaginal mesh excision (CD-IIIb). 
Morbidity from the harvest site is summarized in Table 6. Notably, this included swelling (10.5\%), paresthesia that resolved (5.3\%), paresthesia that remained unresolved $(5.3 \%)$, hematoma (5.3\%), and seroma (10.5\%). Notably, there were no complaints of persistent pain and no wound infections.

\section{Discussion}

There is limited literature on autologous fascia lata sacral colpopexy, mostly consisting of small case series with short-term outcomes. ${ }^{12,18}$ This is the first comparison to our knowledge between RSC with autologous fascia lata vs. mesh. Despite some noted limitations, this study demonstrates that RSC with autologous fascia lata has similar shortterm anatomic outcomes to mesh for women with apical POP.

What remains unknown is the long-term durability of autologous fascia lata. Freeze-dried cadaveric fascia lata has previously demonstrated poor 12-month outcomes and poor 5 year outcomes with $62 \%$ success rate compared to $93 \%$ for mesh, however, that analysis was post-hoc and included only 58/100 original subjects. ${ }^{19,20}$ The hypothesis is that cadaveric fascia promotes an immune response that compromises the integrity of the tissue. It is possible that autologous fascia lata may prove more durable given that it does not promote the immune response.

This investigation aimed to capture both anatomic and patient reported outcomes. There was a single anatomic failure within the fascia group with apical prolapse and anterior wall laxity, which will require surgical revision. There were no posterior recurrences in the fascia group, while the mesh group had five posterior failures and a single recurrent cystocele. The recurrent cystocele required revision with an anterior vaginal wall suspension. It is important to note that none of the patients received a concomitant anterior nor posterior repair at the time of SCP, a practice that some follow and that might decrease the compartment specific failure rate. It is also important to note that in a small series like this the concomitant PV sling might support the anterior vaginal wall more effectively than the mesh sling, and both might support better than no concomitant procedure, although slings are not intended to do this. The primary outcome was apical failure, which would not be influenced by concomitant procedures such as sling, anterior 
repair, posterior repair, or enterocele. The compartment specific outcomes, as well as the patient reported outcomes, will be influenced by an unbalanced application of these procedures. For this reason Fisher's exact test was performed to detect bias that might suggest one cohort received compartment specific repairs more often than the other. Those results appear in Table 2.

Our findings are similar to Scott et al. who demonstrated no recurrent apical prolapse, but a $25 \%$ recurrence of anterior vaginal wall prolapse in twelve patients who underwent RSC with autologous fascia lata. ${ }^{12}$ Alternatively, Latini et al reported a $100 \%$ success rate without apical descent at 18 months in a group of 10 women who underwent open sacral colpopexy with autologous fascia lata. ${ }^{5}$ This suggests that RSC with autologous fascia provides comparable tensile strength in the short term for apical fixation to synthetic alternatives. The success rates can be expected to decline with longer follow up, and our intention is to follow the fascia cohort for 5 years and compare directly with the mesh cohort. Furthermore, the data describes will be used for power calculations in the design of a prospective RCT to further explore the hypothesis.

The low rates of serious adverse events associated with both methods is consistent with prior clinical studies. ${ }^{5}$ Our mesh exposure rate of $2.2 \%$ is comparable to the $1.5-8 \%$ noted in a recent review of 13 randomized clinical trials describing sacral colpopexy. ${ }^{4}$ The single patient who suffered a mesh exposure event requested a revision, which was successful. The immediate morbidity of the fascia lata harvest site must be taken into careful consideration and discussed during preoperative counseling. Patients undergoing graft harvest are at risk for site-specific complications including site swelling, numbness, hematoma, and seroma, which has been previously described. ${ }^{12}$ The majority of these were self-limiting and typically resolved with expectant management. No patients demonstrated functional or ambulatory deficits as a result of graft harvest. There was a single incidence of harvest site hematoma requiring transfusion and one DVT requiring anticoagulation. The DVT is troublesome and all fascia lata harvest patients are now given prolonged venous thromboembolism prophylaxis similar to that in lower extremity orthopedic procedures. ${ }^{21}$ Another patient required aspiration of a thigh seroma. However, postoperative seromas are usually small and resolve on their own and as such our group 
discourages intervention. These findings highlight the importance of preoperative counseling and raise the important question of how much harvest site morbidity is acceptable to avoid the risks of synthetic mesh.

Quality of life is integral to guiding treatment and evaluating the treatment outcomes of POP. This work captured patient reported outcomes using the validated IIQ-7 and UDI-6 questionnaires which reflect urinary symptoms, pelvic discomfort, and quality of life measures related to POP. Both groups demonstrated significant improvement in patientreported outcomes postoperatively. The fascia lata group reported higher IIQ-7 scores compared to the mesh group, which parallels an increase in de novo SUI found in this group. However, none of the patients reporting new onset SUI have elected to proceed with sling placement suggesting symptoms were mild.

Interestingly, women that elected to undergo fascial harvest tended to be younger. The reasoning behind this could be two-fold. The younger population may be more concerned regarding the long-term risks of mesh including vaginal erosion and dyspareunia. They also may be more willing to try a new technique and expect a quick recovery despite the extra harvest site incision.

\section{Weaknesses and Limitations}

This work has several weaknesses that should be mentioned. It is a single surgeon, single institution case series which limits the generalizability of the findings. The numbers were small and the follow up was relatively short at 12.1 months. The groups were not evenly matched, the fascia lata group was significantly younger and less parous than the mesh group. This can of course introduce bias. Despite a significant difference in age and parity between the two groups, the preoperative POP-Q measurements, UDI-6, and IIQ-7 responses were similar.

\section{Conclusions}

This is the first prospective comparison of RSC using autologous fascia lata to mesh. Fascia lata RSC appears to have comparable short-term success rates to mesh RSC and acceptable, but higher than expected morbidity related to the graft site with increased 
operative time. The knowledge gained and questions raised underscore the need for a randomized controlled trial. An instructive video describing the technique has been published and the technique is possible for any surgeon familiar with RSC to learn. ${ }^{22}$ 


\section{References}

1. Weber AM, Abrams P, Brubaker L, et al. The standardization of terminology for researchers in female pelvic floor disorders. Int Urogynecol J Pelvic Floor Dysfunct. 2001;12(3):178-86. PubMed PMID: 11451006. Pubmed Central PMCID: PMC2815805. Epub 2001/07/14.

2. Barber MD, Maher C. Epidemiology and outcome assessment of pelvic organ prolapse. Int Urogynecol J. 2013 Nov;24(11):1783-90. PubMed PMID: 24142054. Epub $2013 / 10 / 22$.

3. Wu JM, Hundley AF, Fulton RG, Myers ER. Forecasting the prevalence of pelvic floor disorders in U.S. Women: 2010 to 2050. Obstet Gynecol. 2009 Dec;114(6):1278-83. PubMed PMID: 19935030. Epub 2009/11/26.

4. Costantini E, Brubaker L, Cervigni M, et al. Sacrocolpopexy for pelvic organ prolapse: evidence-based review and recommendations. Eur J Obstet Gynecol Reprod Biol. 2016 Oct;205:60-5. PubMed PMID: 27566224. Epub 2016/08/28.

5. Latini JM, Brown JA, Kreder KJ. Abdominal sacral colpopexy using autologous fascia lata. J Urol. 2004 Mar;171(3):1176-9. PubMed PMID: 14767295. Epub 2004/02/10. eng.

6. Geller EJ, Parnell BA, Dunivan GC. Robotic vs abdominal sacrocolpopexy: 44-month pelvic floor outcomes. Urology. 2012 Mar;79(3):532-6. PubMed PMID: 22386392. Epub 2012/03/06.

7. Ploumidis A, Spinoit AF, De Naeyer G, et al. Robot-assisted sacrocolpopexy for pelvic organ prolapse: surgical technique and outcomes at a single high-volume institution. Eur Urol. 2014 Jan;65(1):138-45. PubMed PMID: 23806518. Epub 2013/06/29. eng.

8. Geller EJ, Siddiqui NY, Wu JM, Visco AG. Short-term outcomes of robotic sacrocolpopexy compared with abdominal sacrocolpopexy. Obstet Gynecol. 2008 Dec;112(6):1201-6. PubMed PMID: 19037026. Epub 2008/11/28. eng. 
9. Flack CK, Monn MF, Patel NB, Gardner TA, Powell CR. National Trends in the Performance of Robot-Assisted Sacral Colpopexy. J Endourol. 2015 Jul;29(7):777-83. PubMed PMID: 25654587. Epub 2015/02/06. eng.

10. Maher C, Feiner B, Baessler K, Glazener CM. Surgical management of pelvic organ prolapse in women. The Cochrane Library [Internet]. 2010 9-8-11; (8).

11. Powell CR, Tachibana I, Eckrich B, Rothenberg J, Hathaway J. Robotic Sacral Colpopexy. J Endourol. 2018 May;32(S1):S111-s6. PubMed PMID: 29774820. Epub 2018/05/19. eng.

12. Scott VCS, Oliver JL, Raz S, Kim JH. Robot-assisted laparoscopic sacrocolpopexy with autologous fascia lata: technique and initial outcomes. Int Urogynecol J. 2019 Nov;30(11):1965-71. PubMed PMID: 30707257. Epub 2019/02/02. eng.

13. Powell CR, Tachibana I, Eckrich B, Rothenberg J, Hathaway J. Securing Mesh with Delayed Absorbable Suture Does Not Increase Risk of Prolapse Recurrence After Robotic Sacral Colpopexy. J Endourol. 2020 Mar 26. PubMed PMID: 32037875. Epub 2020/02/11.

14. FDA. FDA Safety Communication: UPDATE on Serious Complications Associated with Transvaginal Placement of Surgical Mesh for Pelvic Organ Prolapse Silver Spring, MD: United States Food \& Drug Administration; 2011 [cited 2012 3/11/12]. Update on the FDA black box warning on vaginal mesh]. Available from: http://www.fda.gov/MedicalDevices/Safety/AlertsandNotices/ucm262435.htm.

15. Harvey MA, Kristjansson B, Griffith D, Versi E. The Incontinence Impact Questionnaire and the Urogenital Distress Inventory: a revisit of their validity in women without a urodynamic diagnosis. Am J Obstet Gynecol. 2001 Jul;185(1):25-31. PubMed PMID: 11483899.

16. Bump RC, Hurt WG, Theofrastous JP, et al. Randomized prospective comparison of needle colposuspension versus endopelvic fascia plication for potential stress incontinence prophylaxis in women undergoing vaginal reconstruction for stage III or IV pelvic organ prolapse. The Continence Program for Women Research Group. Am J Obstet Gynecol. 1996 Aug;175(2):326-33; discussion 33-5. PubMed PMID: 8765249. Epub 1996/08/01. eng. 
17. Clavien PA, Barkun J, de Oliveira ML, et al. The Clavien-Dindo classification of surgical complications: five-year experience. Ann Surg. 2009 Aug;250(2):187-96. PubMed PMID: 19638912. Epub 2009/07/30.

18. Chaus FM, Funk JT, Pangilinan J, Lin FC, Twiss CO. Total Autologous Fascia Lata Anterior and Apical Pelvic Organ Prolapse Repair: A New Technique and Initial Experience. Urology. 2020 Mar;137:190-5. PubMed PMID: 31883878. Epub 2019/12/31. eng.

19. Culligan PJ, Blackwell L, Goldsmith LJ, Graham CA, Rogers A, Heit MH. A randomized controlled trial comparing fascia lata and synthetic mesh for sacral colpopexy. Obstet Gynecol. 2005 Jul;106(1):29-37. PubMed PMID: 15994614. Epub 2005/07/05.

20. Tate SB, Blackwell L, Lorenz DJ, Steptoe MM, Culligan PJ. Randomized trial of fascia lata and polypropylene mesh for abdominal sacrocolpopexy: 5-year follow-up. Int Urogynecol J. 2011 Feb;22(2):137-43. PubMed PMID: 20798922. Epub 2010/08/28. eng.

21. Forster R, Stewart M. Anticoagulants (extended duration) for prevention of venous thromboembolism following total hip or knee replacement or hip fracture repair. Cochrane Database Syst Rev. 2016 Mar 30;3:CD004179. PubMed PMID: 27027384. Epub 2016/03/31.

22. Tachibana I, Pickhardt M, Powell C. Robotic Sacral Colpopexy with Fascia Lata for Pelvic Organ Prolapse. Journal of Endourology- Videourology. 20194 Jun 2019(33):3. Epub 4 Jun 2019. 
Table 1: Demographics, Baseline Pelvic Measurements, and Urinary Symptoms

\begin{tabular}{|c|c|c|c|}
\hline Variable & $\operatorname{Fascia}(N=19)$ & $\operatorname{Mesh}(N=45)$ & $P$-value \\
\hline Age at Sacral Colpopexy ${ }^{a}$ & $59(41-91)$ & $68(35-78)$ & 0.01 \\
\hline \multicolumn{4}{|l|}{ Race } \\
\hline White & $16(84.2 \%)$ & $41(91.1 \%)$ & NS \\
\hline Black & $3(15.8 \%)$ & $4(8.9 \%)$ & \\
\hline $\mathrm{BMI}^{\mathrm{a}}$ & $26(18-34)$ & $29(20-45)$ & NS \\
\hline Diabetes & $5(26.3 \%)$ & $1(2.2 \%)$ & 0.01 \\
\hline Current Smoker & 0 & $3(6.7 \%)$ & NS \\
\hline Gravidity $^{\mathrm{a}}$ & $2.3(0-6)$ & $3.3(1-8)$ & 0.03 \\
\hline Parity $^{\mathrm{a}}$ & $2.1(0-4)$ & $3.0(1-7)$ & 0.02 \\
\hline Cesarean Deliveries $^{a}$ & $0.2(0-4)$ & $0.1(0-4)$ & NS \\
\hline Vaginal Deliveries $^{a}$ & $1.8(0-4)$ & $2.9(1-7)$ & 0.01 \\
\hline Prior Hysterectomy & $7(36.8 \%)$ & $22(48.9 \%)$ & NS \\
\hline Prior SUI/POP Surgery & $3(15.8 \%)$ & $13(28.9 \%)$ & NS \\
\hline Voids/24hours ${ }^{b}$ & $7.9 \pm 4.3$ & $15.9 \pm 19.3$ & NS \\
\hline Pads/24hours ${ }^{\text {b }}$ & $1.2 \pm 1.3$ & $1.3 \pm 1.8$ & NS \\
\hline Urge Incontinence & $9(47.4 \%)$ & $16(35.6 \%)$ & NS \\
\hline Stress Incontinence & $7(36.8 \%)$ & $9(20.0 \%)$ & NS \\
\hline Postvoid Residual (mL) ${ }^{b}$ & $51.1 \pm 66.9$ & $62.6 \pm 81.9$ & NS \\
\hline UDI- $6^{b}$ & $11 \pm 4.4$ & $10 \pm 4.5$ & NS \\
\hline $11 Q-7^{b}$ & $11 \pm 8.4$ & $11 \pm 7.4$ & NS \\
\hline \multicolumn{4}{|l|}{ POPQ Value ${ }^{c}$} \\
\hline $\mathrm{Aa}$ & 1 (0 to 3 ) & $0(-3$ to 3$)$ & NS \\
\hline $\mathrm{Ba}$ & $1.5(0$ to 6$)$ & $2(-2$ to 8$)$ & NS \\
\hline Ap & $-1.5(-3$ to 0$)$ & $-2(-3$ to 3$)$ & NS \\
\hline $\mathrm{Bp}$ & $-1(-3$ to 1$)$ & $-1(-3$ to 3$)$ & NS \\
\hline C & $-5(-8$ to -2$)$ & $-5(-8$ to 5$)$ & NS \\
\hline $\mathrm{GH}$ & 3 (2 to 5$)$ & 3 (3 to 5 ) & NS \\
\hline PB & 3 (2 to 3$)$ & 3 (1 to 5$)$ & NS \\
\hline
\end{tabular}




\begin{tabular}{c|c|c|c}
\hline TVL & $8(6$ to 10$)$ & $8(6$ to 11$)$ & NS \\
POPQ Stage $^{c}$ & $3(2$ to 4$)$ & $3(2$ to 4$)$ & NS \\
Stage 0 & 0 & 0 & \\
Stage 1 & 0 & 0 & \\
Stage 2 & $9(47.4 \%)$ & $17(37.8 \%)$ & \\
Stage 3 & $10(52.6 \%)$ & $24(53.3 \%)$ & \\
Stage 4 & 0 & $4(8.9 \%)$ & \\
\hline
\end{tabular}

BMI, body mass index; SUI/POP, stress urinary incontinence/pelvic organ prolapse; UDI-6, Urogenital Distress Inventory-6; IIQ-7, Incontinence Impact Questionnaire-7; NS, not

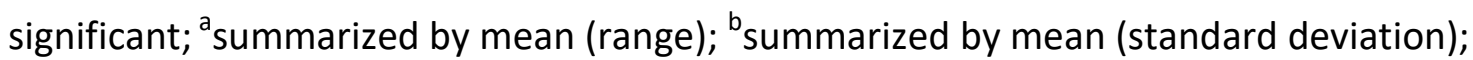
csummarized by median (range) 
Table 2: Operative Details

\begin{tabular}{|c|c|c|c|}
\hline Variable & Fascia $(N=19)$ & Mesh $(N=45)$ & P-value \\
\hline Operative Time $(\min )^{a}$ & $288(210-365)$ & $237(161-326)$ & $<0.001$ \\
\hline Fascia Harvest Time $(\min )^{a}$ & $25(18-40)$ & NA & \\
\hline Estimated Blood Loss (mL) ${ }^{a}$ & $33(20-52)$ & $28(10-80)$ & 0.11 \\
\hline Length of Stay (Days) & $1(0-4)$ & $1(0-3)$ & 0.38 \\
\hline \multicolumn{4}{|l|}{ Concomitant Supracervical } \\
\hline Hysterectomy & $8(42 \%)$ & $16(36 \%)$ & 0.78 \\
\hline Concomitant total hysterectomy & $1(5 \%)$ & $3(7 \%)$ & 1 \\
\hline Concomitant mid urethral sling & $0(0 \%)$ & $8(18 \%)$ & 0.09 \\
\hline Concomitant fascia sling & $6(32 \%)$ & $0(0 \%)$ & $<0.001$ \\
\hline Concomitant anterior repair & $0(0 \%)$ & $0(0 \%)$ & \\
\hline Concomitant posterior repair & $0(0 \%)$ & $0(0 \%)$ & \\
\hline Concomitant enterocele repair & $18(95 \%)$ & $40(89 \%)$ & 0.66 \\
\hline Required Catheterization POD1 & $5(26 \%)$ & $4(9 \%)$ & 0.11 \\
\hline
\end{tabular}

POD1, postoperative day one; ${ }^{a}$ summarized by mean (range); ${ }^{b}$ summarized by median (range) 
Table 3: Combined Outcomes for Fascia Lata

\begin{tabular}{|c|c|c|c|}
\hline Variable & Preoperative & Postoperative & $\begin{array}{c}P_{-} \\
\text {value }\end{array}$ \\
\hline UDI- $6^{\mathrm{a}}$ & $10 \pm 4.5$ & $4.3 \pm 3.8$ & 0.004 \\
\hline $11 \mathrm{Q}-7^{\mathrm{a}}$ & $11 \pm 7.4$ & $2.2 \pm 5.1$ & 0.02 \\
\hline POPQ Value & & & \\
\hline $\mathrm{Aa}$ & 1 (0 to 3$)$ & $-3(-3$ to 2$)$ & $<0.001$ \\
\hline $\mathrm{Ba}$ & 1.5 (0 to 6$)$ & $-3(-3$ to -3$)$ & $<0.001$ \\
\hline Ap & $-1.5(-3$ to 0$)$ & $-3(-3$ to -1$)$ & $<0.001$ \\
\hline $\mathrm{Bp}$ & $-1(-3$ to 1$)$ & $-2(-3$ to 0$)$ & 0.01 \\
\hline C & $-5(-8$ to -2$)$ & $-8(-10$ to -6$)$ & $<0.001$ \\
\hline $\mathrm{GH}$ & $3(2$ to 5$)$ & 3 (3 to 4$)$ & 0.33 \\
\hline PB & 3 (2 to 3 ) & 3 (3 to 3 ) & 0.16 \\
\hline TVL & 8 (6 to 10$)$ & $9(7$ to 10$)$ & 0.13 \\
\hline POPQ Stage & $3(2$ to 4$)$ & 1 (0 to 3$)$ & 0.0001 \\
\hline Stage 0 & 0 & $4(21.1 \%)$ & \\
\hline Stage 1 & 0 & $6(31.6 \%)$ & \\
\hline Stage 2 & 9 (47.4\%) & $8(42.1 \%)$ & \\
\hline Stage 3 & $10(52.6 \%)$ & $1(5.2 \%)$ & \\
\hline Stage 4 & 0 & 0 & \\
\hline
\end{tabular}

UDI-6, Urogenital Distress Inventory-6; IIQ-7, Incontinence Impact Questionnaire-7;

${ }^{a}$ summarized by mean(standard deviation); ${ }^{b}$ summarized by median (range) 
Table 4: Combined Outcomes for Mesh

\begin{tabular}{|c|c|c|c|}
\hline Variable & Preoperative & Postoperative & $P$-value \\
\hline UDI- $6^{\mathrm{a}}$ & $11 \pm 4.4$ & $5.5 \pm 3.0$ & $<0.001$ \\
\hline $11 Q-7^{a}$ & $11 \pm 8.4$ & $4.9 \pm 4.6$ & $<0.001$ \\
\hline \multicolumn{4}{|l|}{ POPQ Value } \\
\hline $\mathrm{Aa}$ & 0 (-3 to 3$)$ & $-3(-3$ to 0$)$ & $<0.001$ \\
\hline $\mathrm{Ba}$ & $2(-2$ to 8$)$ & $-2(-3$ to 0$)$ & $<0.001$ \\
\hline$A p$ & $-2(-3$ to 3$)$ & $-2(-3$ to 2$)$ & 0.002 \\
\hline $\mathrm{Bp}$ & -1 (-3 to 3$)$ & $-2(-3$ to 2$)$ & 0.007 \\
\hline C & -5 (-8 to 5$)$ & $-8(-10$ to -5$)$ & $<0.001$ \\
\hline $\mathrm{GH}$ & 3 (3 to 5$)$ & 3 (2 to 5$)$ & 0.01 \\
\hline PB & 3 (1 to 5$)$ & 3 (2 to 5$)$ & NS \\
\hline TVL & $8(6$ to 11$)$ & 8 (5 to 10$)$ & NS \\
\hline POPQ Stage $e^{b}$ & $3(2$ to 4$)$ & 2 (0 to 3$)$ & $<0.001$ \\
\hline Stage 0 & 0 & 5 (11.1\%) & \\
\hline Stage 1 & 0 & 7 (15.6\%) & \\
\hline Stage 2 & 17 (37.8\%) & 32 (71.1\%) & \\
\hline Stage 3 & 24 (53.3\%) & $1(2.2 \%)$ & \\
\hline Stage 4 & 4 (8.9\%) & 0 & \\
\hline
\end{tabular}

UDI-6, Urogenital Distress Inventory-6; IIQ-7, Incontinence Impact Questionnaire-7; NS, not significant; ${ }^{a}$ summarized by mean (standard deviation); ${ }^{b}$ summarized by median (range) 
Table 5: Postoperative Comparison Fascia Lata vs. Mesh

\begin{tabular}{|c|c|c|c|}
\hline Variable & Fascia $(N=19)$ & Mesh $(N=45)$ & P-value \\
\hline Voids/24hours ${ }^{a}$ & $4.4 \pm 2.0$ & $6.6 \pm 3.6$ & 0.03 \\
\hline Pads/24hours ${ }^{a}$ & $1.2 \pm 1.3$ & $0.66 \pm 1.7$ & 0.20 \\
\hline Urge Incontinence & $5(26.3 \%)$ & $8(17.8 \%)$ & 0.74 \\
\hline Stress Incontinence & $4(21.1 \%)$ & $1(2.2 \%)$ & 0.02 \\
\hline Postvoid Residual $(\mathrm{mL})^{a}$ & $30 \pm 74.2$ & $31 \pm 41.5$ & 0.96 \\
\hline UDI- $6^{a}$ & $5.5 \pm 3.0$ & $4.3 \pm 3.8$ & 0.25 \\
\hline$\| \mathrm{QQ}-7^{\mathrm{a}}$ & $4.9 \pm 4.6$ & $2.2 \pm 5.1$ & 0.05 \\
\hline POPQ Value ${ }^{b}$ & & & \\
\hline $\mathrm{Aa}$ & $-3(-3$ to 2$)$ & $-3(-3$ to 0$)$ & 0.49 \\
\hline $\mathrm{Ba}$ & $-3(-3$ to -3$)$ & $-2(-3$ to 0$)$ & 0.41 \\
\hline Ap & $-3(-3$ to -1$)$ & $-2(-3$ to 2$)$ & 0.04 \\
\hline $\mathrm{Bp}$ & $-2(-3$ to 0$)$ & $-2(-3$ to 2$)$ & 0.13 \\
\hline C & $-8(-10$ to -6$)$ & $-8(-10$ to -5$)$ & 0.22 \\
\hline $\mathrm{GH}$ & 3 (3 to 4$)$ & 3 (2 to 5$)$ & 0.62 \\
\hline PB & 3 (3 to 3 ) & $3(2$ to 5$)$ & 0.65 \\
\hline TVL & 9 (7 to 10$)$ & 8 (5 to 10$)$ & 0.14 \\
\hline POPQ Stage ${ }^{b}$ & 1 (0 to 3$)$ & 2 (0 to 3 ) & 0.12 \\
\hline Stage 0 & $4(21.1 \%)$ & $5(11.1 \%)$ & \\
\hline Stage 1 & $6(31.6 \%)$ & 7 (15.6\%) & \\
\hline Stage 2 & $8(42.1 \%)$ & $32(71.1 \%)$ & \\
\hline Stage 3 & $1(5.2 \%)$ & $1(2.2 \%)$ & \\
\hline Stage 4 & 0 & 0 & \\
\hline Recurrent Cystocele & $1(5.3 \%)$ & $1(2.2 \%)$ & 0.45 \\
\hline Recurrent Rectocele & 0 & 5 (11.1\%) & 0.31 \\
\hline Recurrent Apical Descent & $1(5.3 \%)$ & 0 & 0.30 \\
\hline Follow-Up (Months) ${ }^{a}$ & \multicolumn{2}{|c|}{$12.1 \pm 8.7$} & \\
\hline
\end{tabular}


UDI-6, Urogenital Distress Inventory-6; IIQ-7, Incontinence Impact Questionnaire-7; ${ }^{a}$ summarized by mean (standard deviation); ${ }^{b}$ summarized by median (range) 
Table 6: Site-Specific Complications Associated with Fascia Lata Harvest

\begin{tabular}{l|c}
\hline Complication & Number of Patients $(N=19)$ \\
\hline Swelling & $2(10.5 \%)$ \\
DVT & $1(5.3 \%)$ \\
Pain & 0 \\
Infection & 0 \\
Paresthesia (Resolved) & $1(5.3 \%)$ \\
Paresthesia (Unresolved) & $1(5.3 \%)$ \\
Hematoma & $1(5.3 \%)$ \\
Seroma & $2(10.5 \%)$ \\
\hline
\end{tabular}

DVT, deep vein thrombosis 


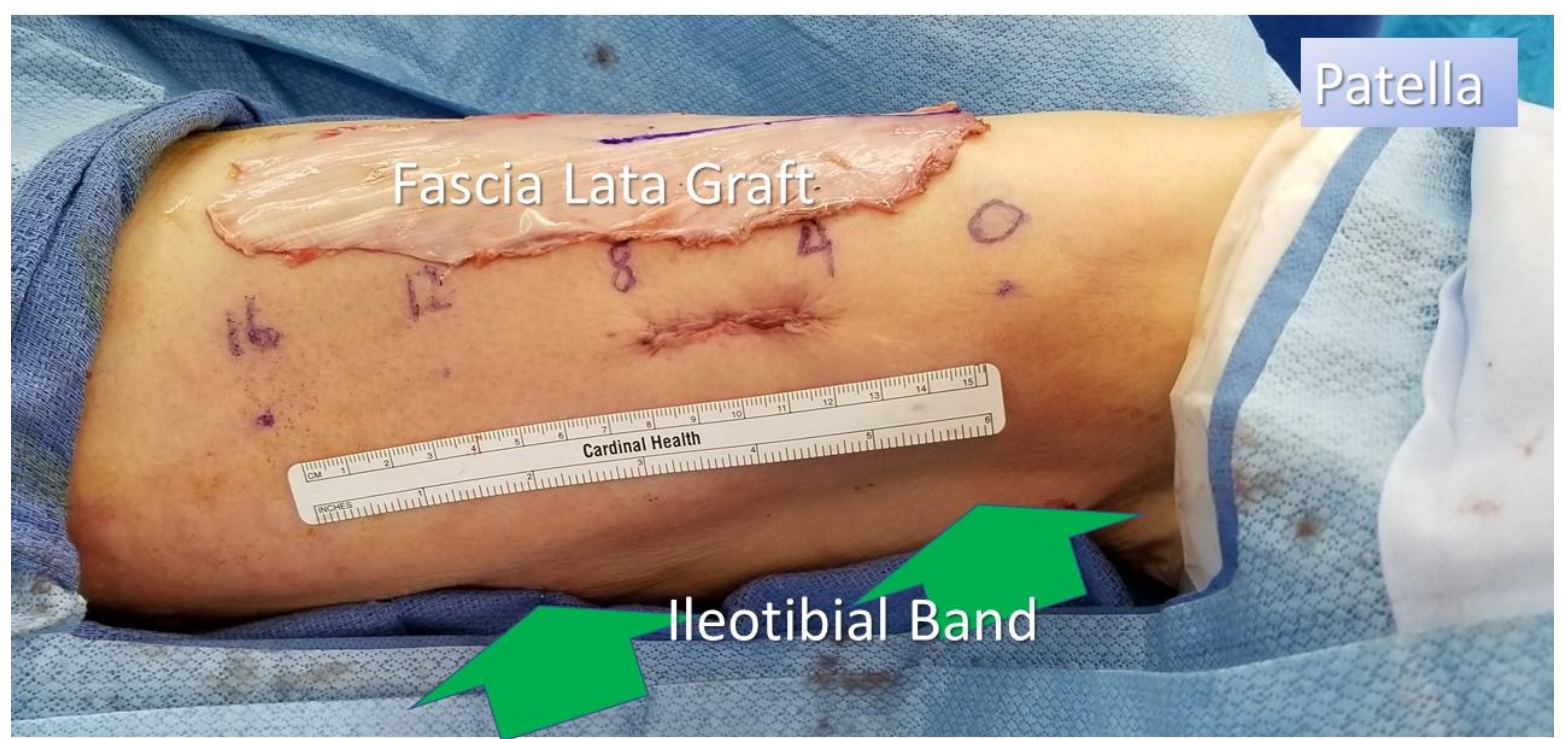

Figure 1: Sample harvest site and fascia lata graft. This figure demonstrates a $16 \mathrm{~cm}$ fascia lata graft obtained through a $4 \mathrm{~cm}$ skin incision. The graft is harvested anterior to the iliotibial band. 


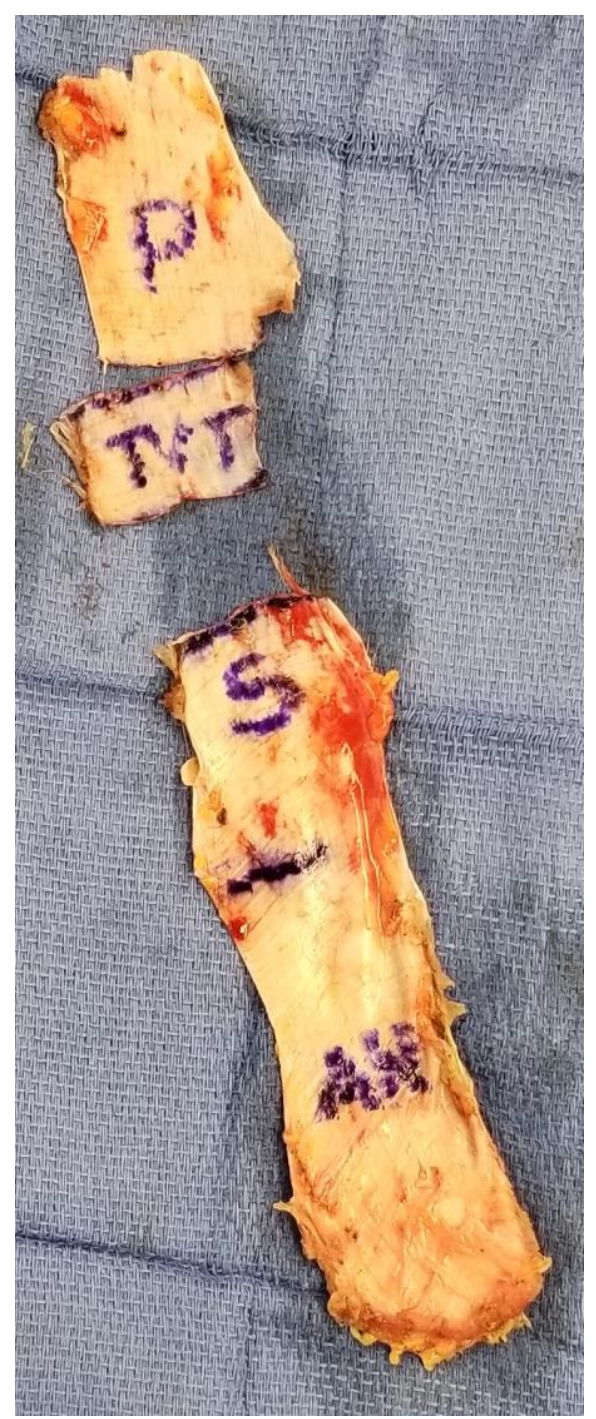

Figure 2: Sample fascia harvest. Multiple fascial pieces can be obtained from a single incision and harvest of fascia lata. This demonstrates fascia configured for posterior (P), anterior (AN), sacral (S), and pubovaginal sling (TVT). 


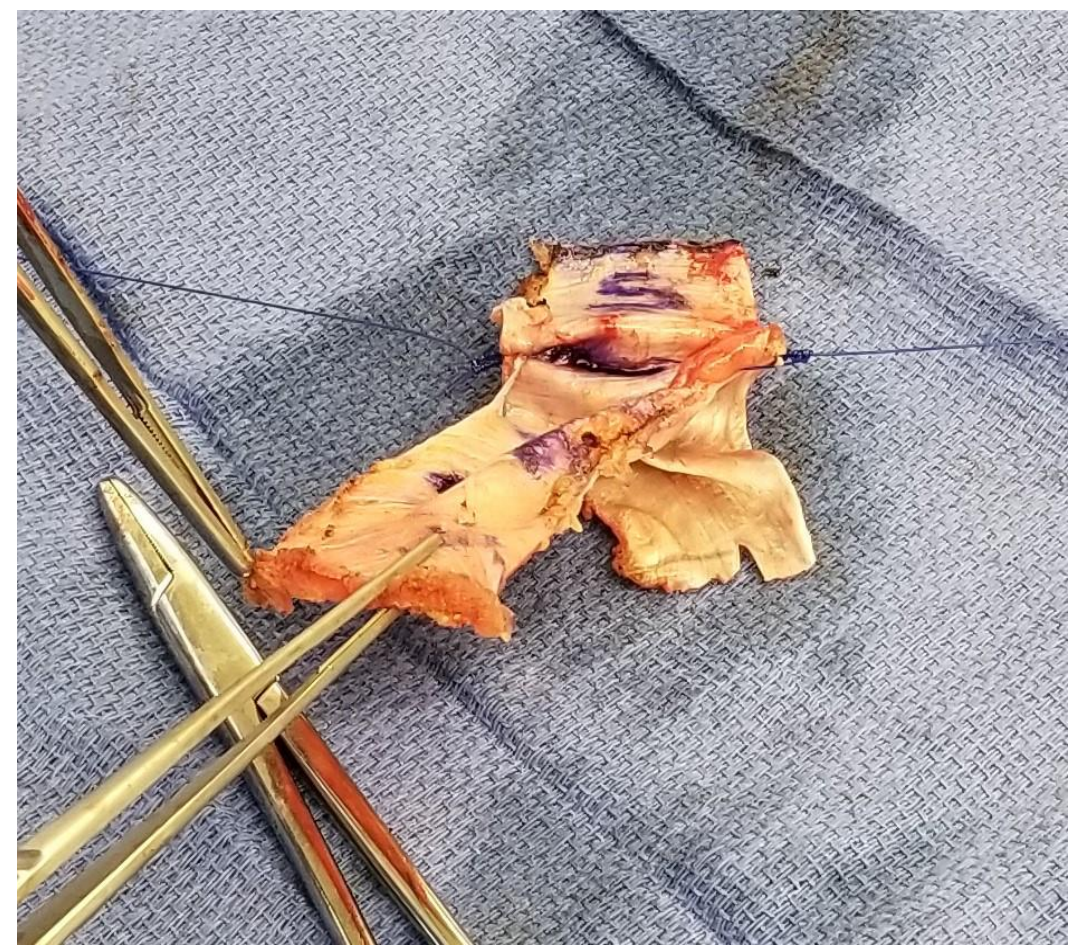

Figure 3: $Y$ configuration. The fascia graft is configured into a $Y$ configuration using 0 -nonabsorbable monofilament. Two leaflets will be secured to the anterior and posterior vagina, while the segment labeled " $\mathrm{S}$ " will be secured to the sacrum. 
Abbreviations Used

RSC = robotic sacral colpopexy

POP-Q = Pelvic Organ Prolapse Quantification

$\mathrm{CD}=$ Clavien-Dindo

$\mathrm{POP}=$ pelvic organ prolapse

FDA $=$ Food and Drug Administration

FPMRS = Female Pelvic Medicine and Reconstructive Surgeon

UDI-6 = Urogenital Distress Inventory-6

IIQ-7 = Incontinence Impact Questionnaire-7 